\title{
$\mathfrak{s} \mathfrak{n} \mathfrak{h} \mathfrak{a} \mathfrak{l} \mathfrak{t}$
}

\author{
Eritte $\mathfrak{A}$ bteilung. $1467-1476$. \\ Die burgundifde Geirat.
}

Crites Rapitel. S. 3-23. Mittelalter uno Reuzeit. Die Anfänge mo: derner Stantenbiloung im 15. Sahrhundert. Deutide Buftönde. 2hbrenzung des Mittelalter uno ber Menzeit $3-6$, Neuvronung der inter=

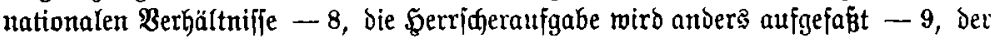
Fürit nad Eomnnes uno Machiabelli - 11, Redjte und Pflidten des netten

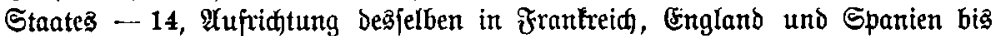

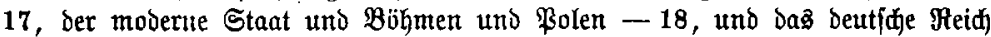

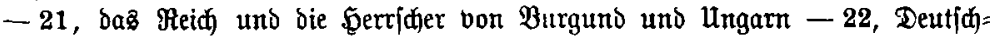
Iand uno D̈pterreid -28 .

3weites Sapitel. 5. 23-37. Der Rirdenftreit in Babmen nad der

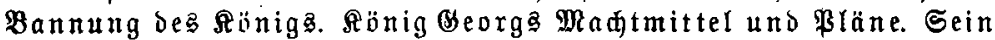

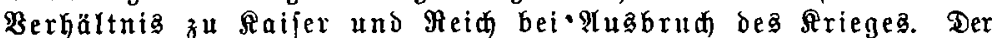
zweite Sulfitenfrieg bes 15. Jahrhunderts uns jeine Bedeutung - 24, Macht=

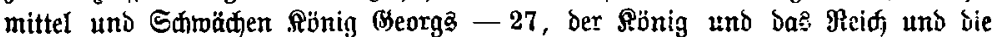

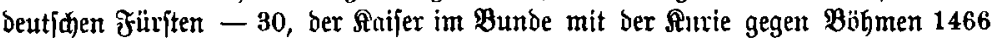
- 32, Beziehungen zwifhen Rörig (Seorg und Saijer Friedrich vom Spătjahr 1466 bis zum Februar $1467-37$.

Drittes Sapitel. 5. 37-69. Das Reid und ber beutide Rorben. Rolen und der Deutidorben. Sdleswig= bon Dänemart. Die Bommeride Erbfolgefrage. Das Reid und ber

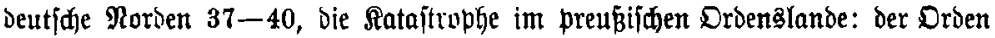
und jeine Randjtände bis $1455-41$, filfeleiftung ber $\mathfrak{B}$ olen für bie Stände

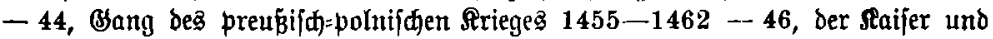

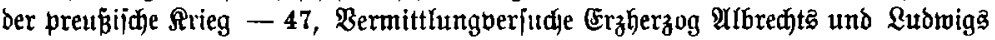

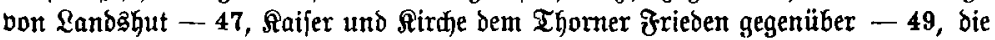
Erwerbung Đolfteing bon Seiten Däuemarks: Erbebung (Sraf Chriftianz von Oldenburg auf den dänifłen Thron (28. September 1448) - 50, Charafteriftif Rönig Chriftians I. - 52, feine Stellung zum Reidye - 52, Ehrifitian viro

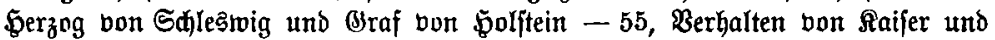

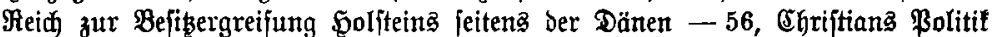
bis 1473 - 57, utriprung uno Berlauf des pommeriden Streites 1464 -59, Stellung des Saifers -59, S(bmadungen des Saifers und ber Martgrafen

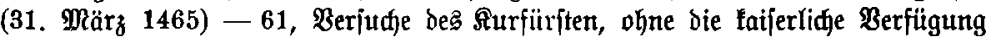
bie Rande zu erwerben - 63, der Berlag von Soldin (21. Jan. 1466) - 64,

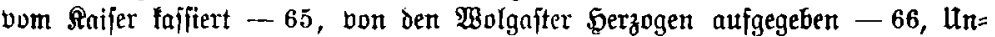
wille ber Minrfgrafen gegent Den Saifer -67 , Bommern für fie verloren -68 ,

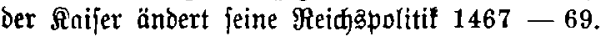

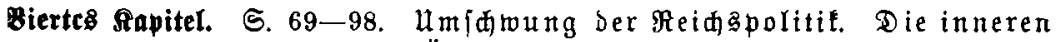
Birren in Bänen und D̈jerreid. Raiferlide und böhmifde $\mathfrak{A} m=$

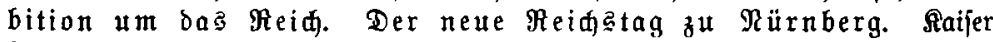
Friedrid nähert fid ben $\mathfrak{B}$ ittelsbađern, Februar $1467-70$, Aufregung bes 


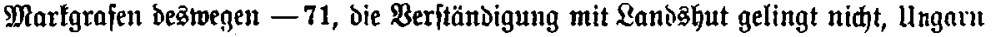

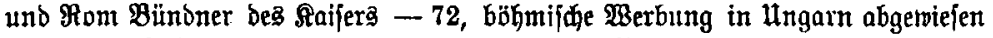
- 75, ber Faftenlanotag (24. F̌ebruar 1467) zu Prag - 77, Ergebniffe - 78, die Bermittelung für Böhmen iu Fom abgewiejen -80 , Betwegungen in ben

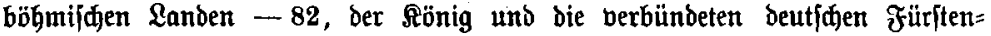

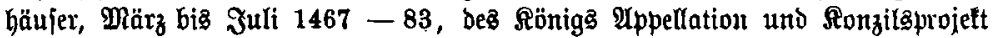

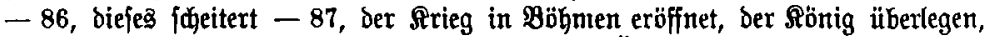

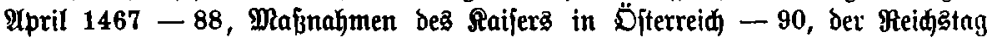

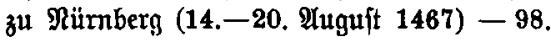

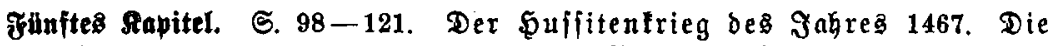

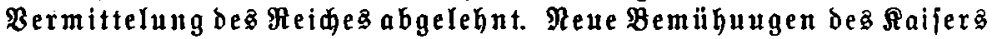

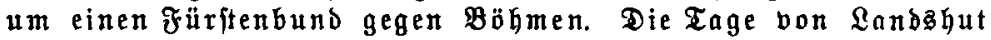

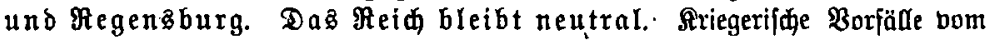
April-Mai 1467 in Böhmen -100, Mapregeln der Furie gegen Bobiebrao

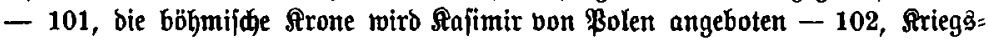

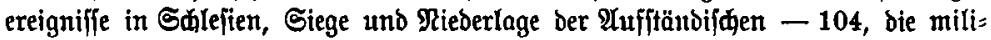

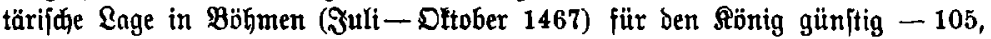
Bolen und Frantrei亩 ihm geneigt - 106, bie Bermittelungånnträge ber beutidjen Füriten vor bem Rönige ( $Y$ uguft 1467) - 108, werben abgelehnt Dltober

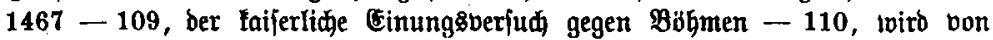
Bayern = Ranbshut unteritït -112 , aber dennod in Regenşburg (18. Nou.)

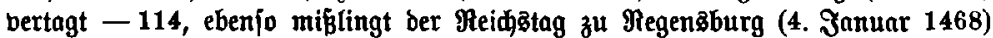

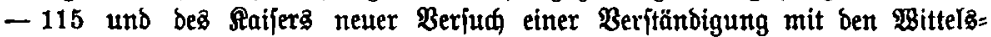

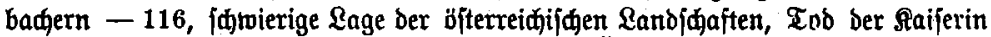

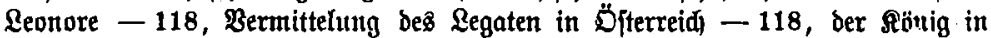
Böbmen in Spätjahre 1467 jür bie \&iga günjitiger - 120, Errgebniffe - 121 .

Sediftes Sapitel. S. 121-145. Burgund und ungarn gegenüber bemböh= mifden Rriege. Die polnifde Bermittelung und ber Ligatag zu Bres̆lau. Bäpftliđe Berbung am brandenburgiđen bole. Der B̈terreidifde Rrieg und Matthias bon ungarn. Die burgundifde

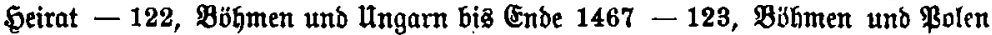
- 125, bie polnijßje Bermittelung im Streite um Böhmen - 129, ber Rigatng

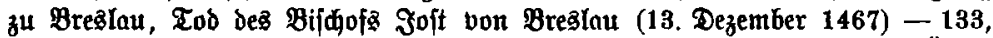

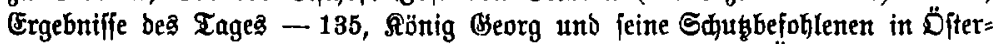

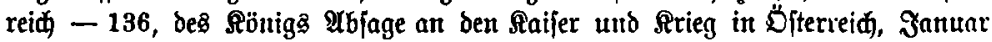

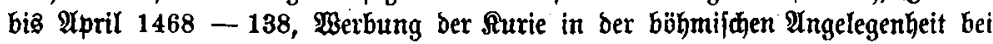

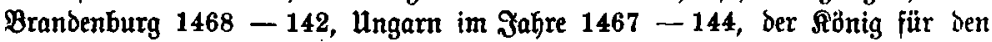

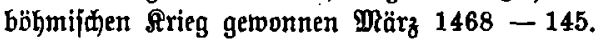

Siebentes Rapitel. S. 145-171. Matthias von ungarn und Beorg von

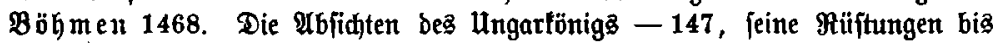
148, Abmadjungen mit ber \&iga und bem Raifer -149 , Werbung am pol=

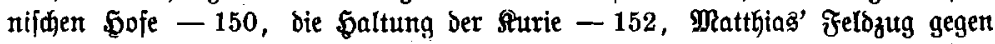

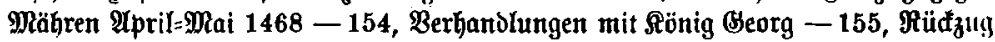

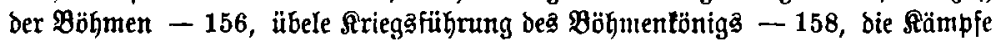
um Trebitich, bie Ungarn erobern die Stabt -159 , Folgen der ungarifđen

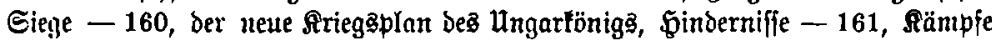

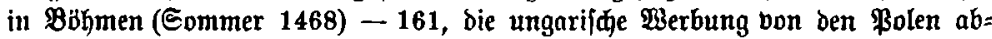

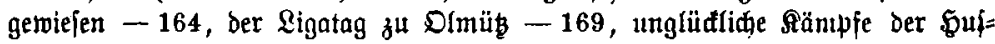




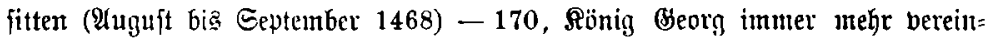
famt -171 .

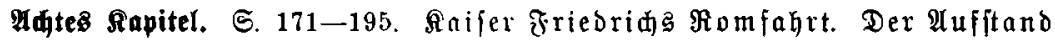

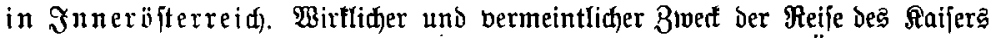

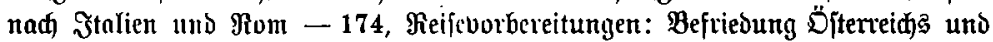

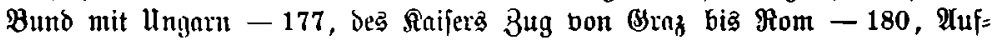
enthalt in Fom (24. Dez. bis 9. Jan.) - 186, Der Faifer in Bettedig - 188, Untergrund bes ïfterreidiijacn OHufitondes (Februar 1469) - 191, Deffen Bwert

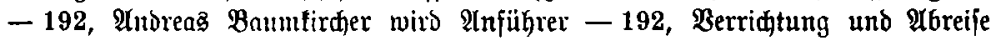
des Faiferż von Bentedig 19. Febr. 1469 - 194, Befämpfung Der $\mathfrak{A}$ ufftändild)cn (F́ebr.-März 1469) - 195.

Reuntes Rapitel. S. 195-220. Das Reid und bie b̈̈hmifde Frage 1468

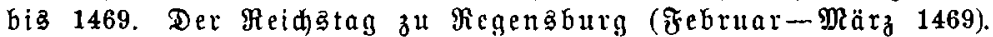
R̈̈nig Matthiag vor Wilemow. Der Friebe zivifaen den Fïnigen und feite Folgen. Das Reid und Bibmen 1468 - 198, Die Früritenver= fommlungen zu Crfurt, Spener und Landhut - 199, Dez Reid)steg zu Regens=

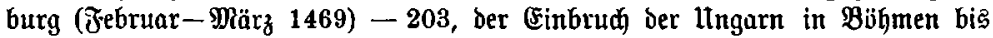
206, bie Berbanolungen und $\mathfrak{A}$,

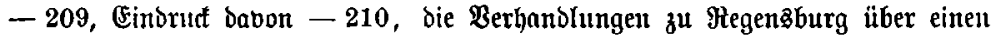

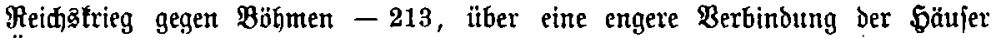
S̈fterreich, Bayern, Sadjfen und Branbenburg - 214, bie Regaten, Rafimir bou

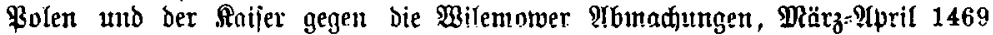

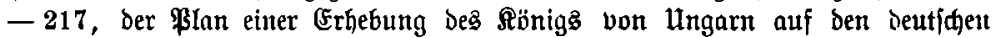

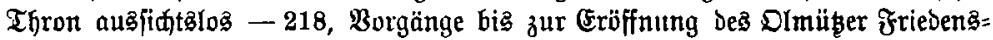
tage (7. Upril 1469) -220.

Behntes Rapitel. 5. 220-241. Die Olmü̈er Friedeñverhanblungen.

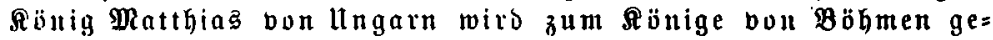
währt. Bolitif bes llngartänigs. Der Braqer Junilandag. Der Rampf in Juneristerreid. Sünig Matthias verftüudigt fid wieder mit bem Raijer. Neue $\mathfrak{B}$ andrung ber Dinge. Die Ormüher Friebensz= berbansluntgent fübren nur zur Berlängenung ber 2 affenmthe bis 1 1. Jan. 1470

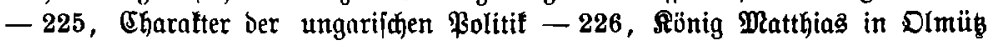
zum böhmifłen $\Re$ önige gewählt (3. Mai 1469) - 227, jeine neue Berhandlung unit Rafimir von Bolen - 229, Einflup Der Olmilizer $\mathfrak{W a h l}$ auf ben (Santg ber

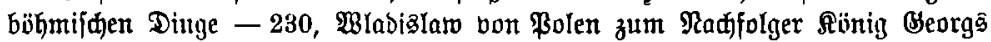
beftimmt -- 231, (Einbrud) der Türfen in Rrain - 232, bie Rurie gegen die

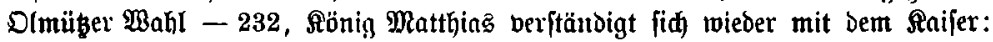

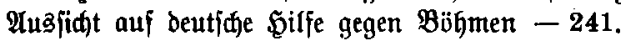

Giftes Rapitel. S. 241-273. Raifer Friebrid und bie Angelegenheiten bes beutfden Sübens und Befteng. Reue Ziele der Reidspolitil 1470. Der ferzog bon Tirol und die Eibgenoffen. Burgund und Borberöfterreiđ. Die Berträge von Saint Omer, igre Bebeutung und igre eriten Folgen. Saifer Friebrif und bie Fehden in Süb= und

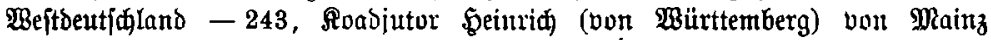

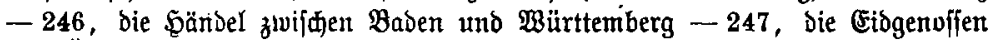
und Öfterreid) -248 , uno Gerzog Sigmuno von Tirol - 250, offene Streit= fälle, ber $\mathfrak{B a l b s h u t e r ~ R r i e g ~ - ~ 2 5 5 , ~ b i e ~ W a r b s ̧ u t e r ~ H i d t u n g ~ ( 2 7 . ~ 2 u g u j t ~ 1 4 6 8 ) ~}$ - 256, Lage bes Gerzogs bon Tirol -257 , feine Entjdlïffe -258 , fein ber= 


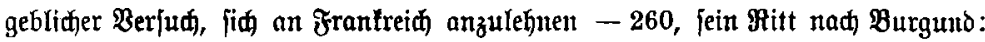

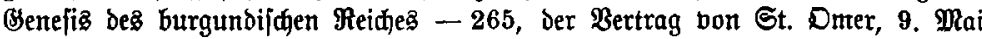

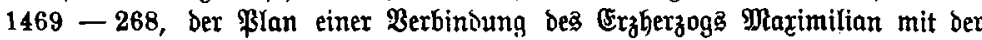
burgundifđen Erbin - 269, Bedeutung Des Beldehenen, Waltung ter neuen

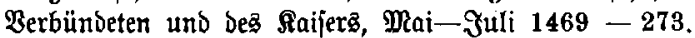

3wolftes Sapitel. S. 274-294. Bedrängte finanzielle Rage des Raifers und ber z̈fterreidifden Erblande. Der Böhmenlönig und das

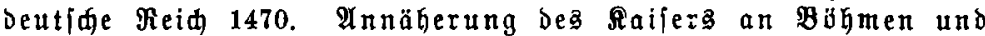
Bolen. Burgund und bie beutfáe Rrone 1470-1471. Bedrängte fitan=

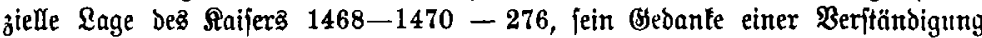
mit $\mathfrak{B a ̈ h m e n ~ - ~ 2 7 7 , ~ B e r h a n o l u n g e n ~ m i t ~ \Re ̈ ̈ n i g ~ M a t t h i a ̨ ~ b o n ~ U n g a r n , ~ \Re o b e m = ~}$

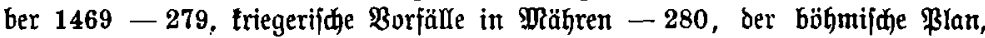

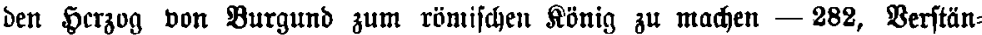

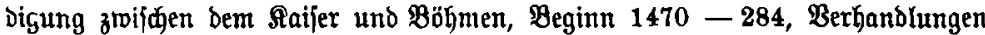
zwijđen Burgund und Tirol über bie Sdmeizerfrage - 286, und iiber bie

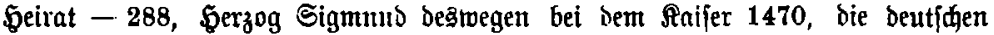

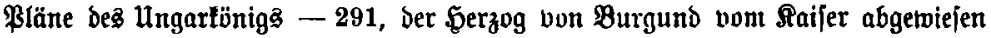
wie ber Rönig bon Ungarn, Beginn 1470 - 294.

Dreizehntes Sapitel. S. 294-322. Die Befriedung ber B̈fterreidifden

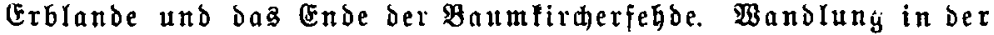
beutiden ßoliti bes Raifers feit 1470. Raifer und Bfalzgraf. Der

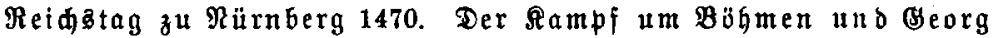

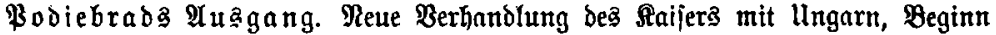

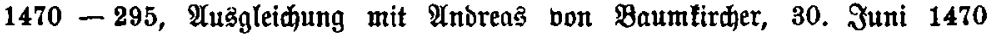

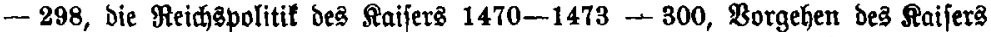

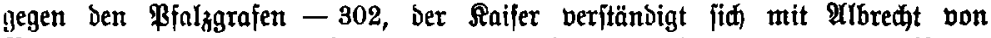
Braubenburg - 303, ber $\Re$ teiđzłtrieg gegen $\mathfrak{B f a l z}$ ungünítig - 304, ber $\mathfrak{R u ̈ r n =}$

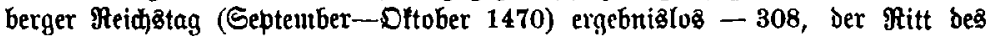

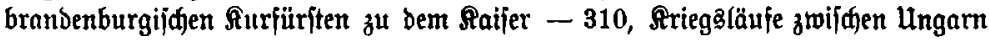

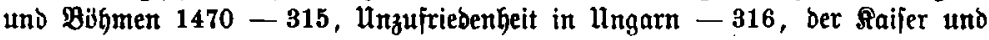
Böhmen $1470-319$, Berhandlungen zwiłten Utngam und Bö̆men - 321, Tos \ünig Beorgà, 22. Măr 1471 - 322 .

Bierzelyntes Rapitel. S. 322-346. Bähmen ná bem bingange Rünig Beorgs. Die Bewerbung berzog albredta bon Sadien um die böhmifae Rrone und bie Bahl

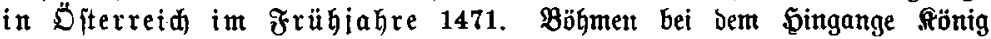

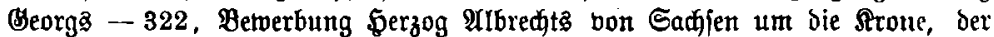

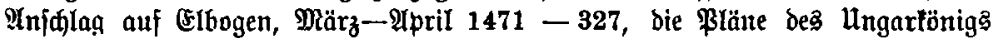
- 329, Die polnifine altion - 330, Det Stillitand von Deutidbrob - 330, Umtriebe zu gunjten Der ßolen - 332, der Lanbtag zu Ruttenberg, 21.-27.

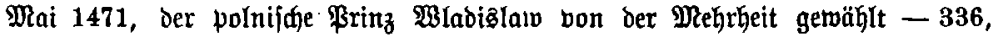

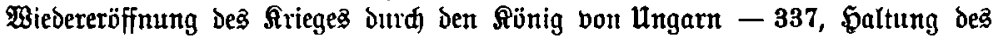

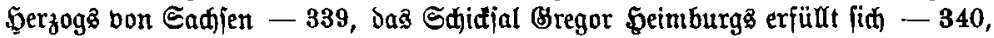

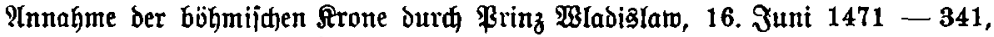

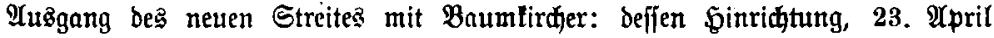
1471 - 344, Bebeutuny bcr That - 345, Des Raijers Bug nat Riegenaburg - 346.

Fluffehntes Sapitel. S. 347-371. Der groß̉e Chriftentag in Megens=

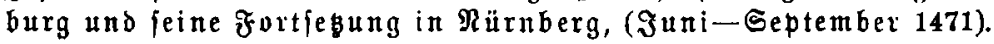




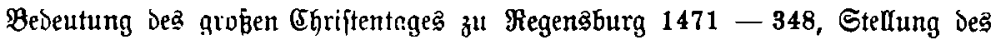
Raijers zll Beginn bes Inges $\mathbf{- 3 4 9}$, bejien $\mathfrak{B}$ ejuher -350 , Beginn der $\mathfrak{B e r}=$ fondlungen, 24. Эuni $-\mathbf{3 5 1}$, bie Berhanolungen über bie faifertiden $\mathfrak{B r o}=$ pofitionen - 353-364: Galtung bes Sarbinallegaten Franz (von Sienta) bia 353, die Befdlüfie des $\mathfrak{I n g e s}$ - 363, die Berhandhung des Raijers mit ben

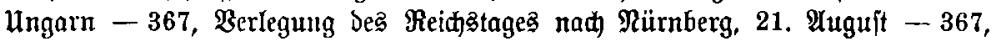

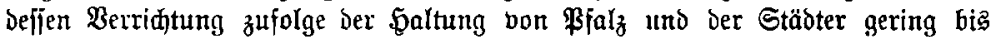

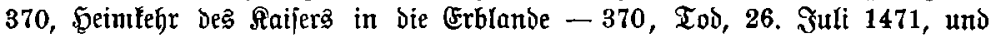
Wiirbigung Bapit $\mathfrak{B a u l s ~ I I . ~ - ~} 371$.

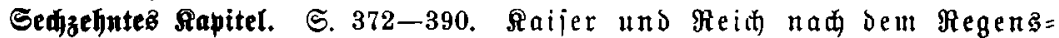
burger Meidstage. Berhandung und Rrieg zwijden den Rönigen bon $\mathfrak{u}$ garn und Bolen. Der ungarifde $\mathfrak{A} u$ fítand und Raifer

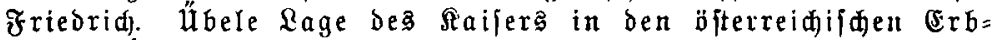

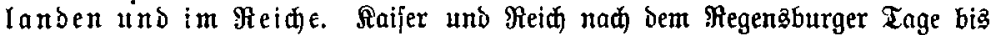

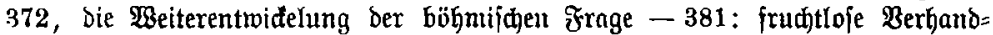

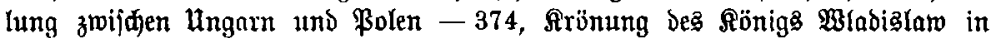
Prag, 22. 2luguft $1471-375$, der erfolglofe (Einbrud Der Polen in Hugarn

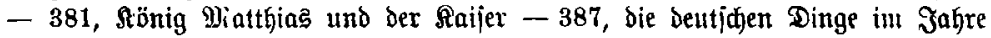
$1471-1472-390$.

Siefzehntes Rapitel. S. 391-421. Sigmund von Tirol and Rarl von Burgund 1470-1473. Die $\mathfrak{B}$ läne, bie burgundifate geirat und bas

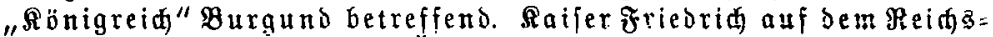

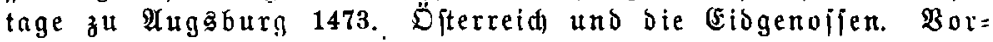
gefdidte ber Fitritenbegegnung zu Trier. Gerzog Sigmunb bon Tirol und Rarl von Burgund 1470-1473 - 390, Berhandlungen über eine Pichtung

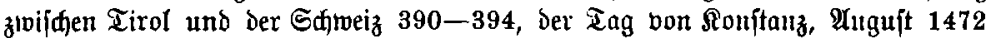

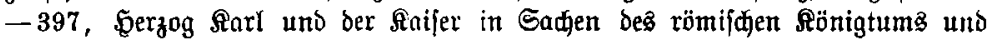

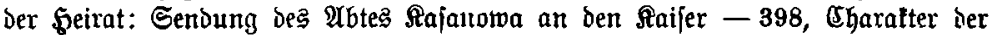

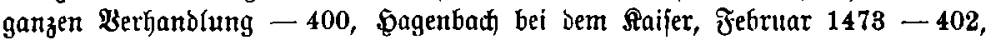

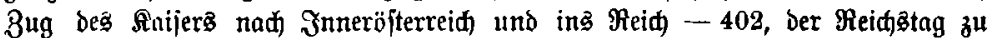

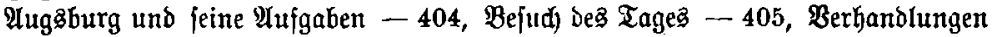

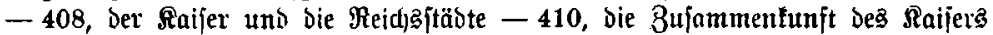

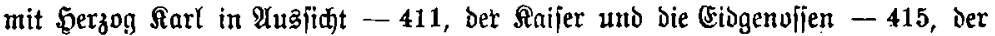

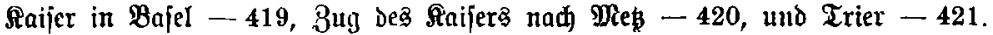

Matzehntes Rapitel. S. 421-442. Ratier Friedrid und Serzog Rarl von Burguno in Trier. Der Streit im Erzftifte $\mathfrak{R} \not \mathfrak{l}$. Sadlage $z u$ Beginn 1474. Raijer Friebrid uno Rarl bon Burguns in Trier, Finzug unt Umgebung ber F̧üriten -424, Charafter ber Berhandlung - 425, Bang Der Berhantblungen - 433, bie Fürrten einig, ferzog farl mit Beldern belehnt

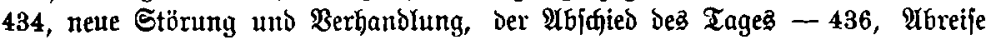

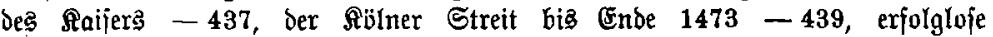

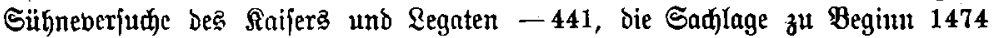
$-442$.

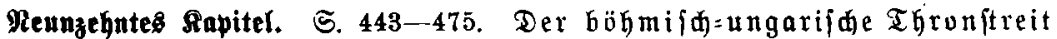
bon 1471-1474. Efriftian I. bon Dänemarf uno Raijer Friedric.

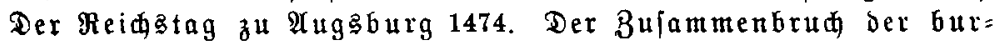
gunoifaen \$errifaft am Dberrhein. Farls oun Burgund Einfall

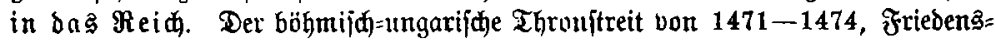
tage zu Reiffe, März 1473, und Troppau, Ruguft $1473-451$, Der Saifer in 


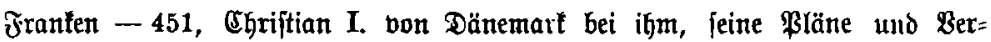

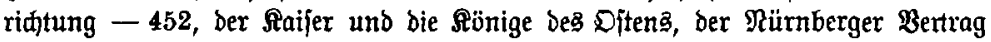

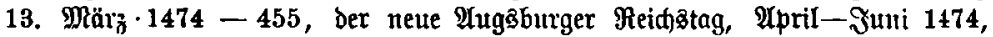
Beju化 Deå Tages - 456, der Raijer gegen bie Stäbte - 458, gegen den $\mathfrak{B j a l z}=$

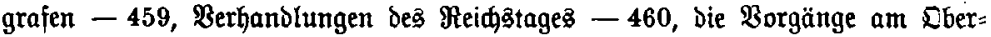
rhein bis zum Pai 1474, bie erwige Richtung, 30. Pärz 1474, mit ben (Fio=

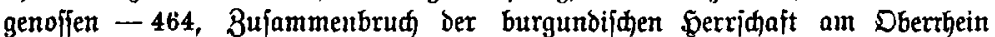

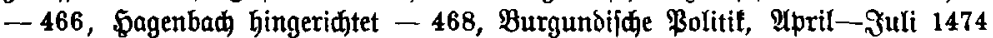

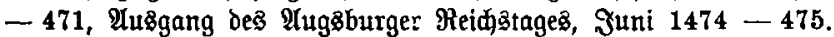

3wanzigftes Rapitel. S. 476-520. Der burgunbijue Finbrud in bas

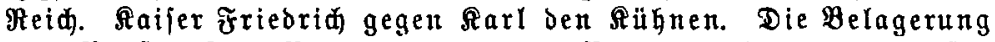

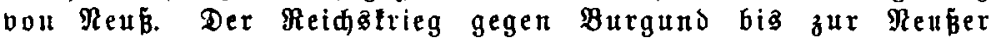

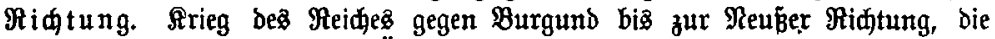

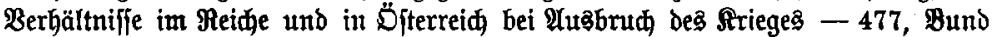
zmifđen Burgund und England - 478, bie Burgunder vor $\mathfrak{N e u ß \beta}$, Juli-Oltober

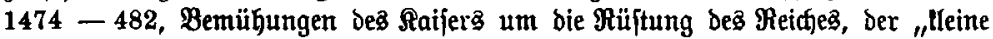

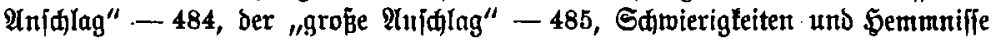

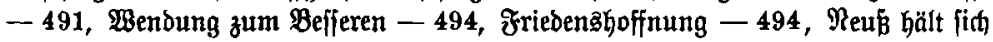

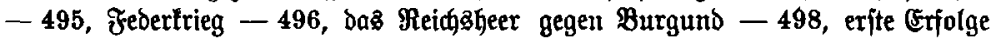

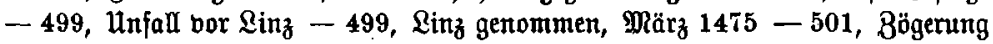
- 503, Berhandlungen - 504, Buno mit Franlreid - 506, Lothringen gegen

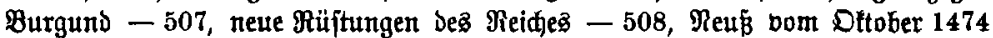

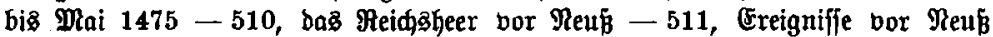

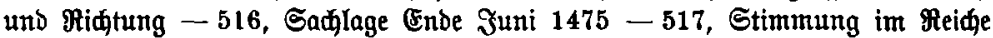

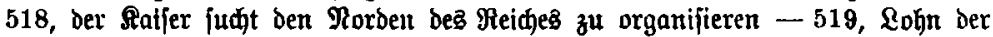
Tapferleit $\mathbf{- 5 2 0}$.

\section{3weite $\mathfrak{A}$ bteilung. $1477-1486$ (1490).}

Das deutiđe Reih im Begenfatge zullngarn. Die römifłe Königswahl Marimilians I.

Cinnnbzwanzigites Sapitel. S. 523-547. Die. Sadlage na出 ber Neuper Ridtung. Der Raijer und ber Difen. Der Raijer und Frantreid nad)

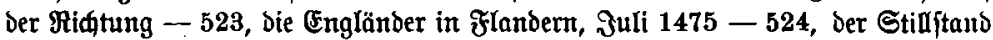
zu Soleuvre, 13. September $1475^{\circ}-526$, ber \$erzog bon Lothringen von Frantreid preisgegeben - 527, die Burgunder gegen Lothringen, Fall bou Rancy - 528, Der Raijer und bie Expedition ber Burgunder gegen Dothringen

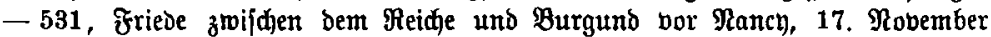

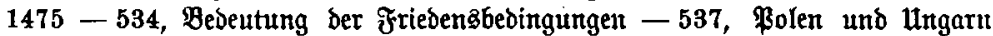
1474-1475 - 538, ber Felozug der $\mathfrak{B b y m e n ~ u n b ~}$ Bolen gegen Saleften, \$erbft $1474-540$, endet unglürtlid -541 , Frièe zu $\mathfrak{B r e s l a u}, 8$. Dezember 1474 -542, Der Saijer tritt bem Brealauer Frieden bei - 543, Bemühungen, Die B̈fterreidiffuen Erblande zu berubigen - 546, brobender neuer Streit -547 .

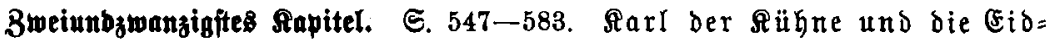
genoffen. Die Sqla bt bei Brandion. Raijer und Derzog. Die

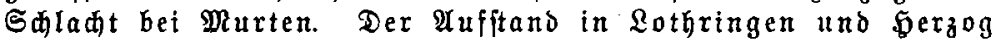

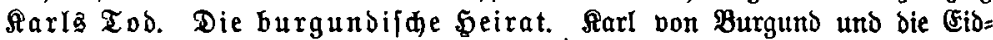
genoffen 1475-1476 - 548, Ieşte Berhandílungen, Januar $1476-551$, die Burgunder gegen Bern im Felve - 552, Safladt bei Grandjon - 554, Folgen

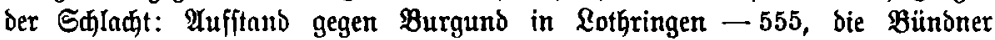

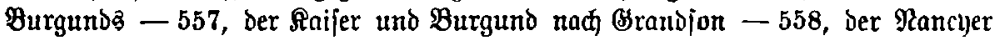




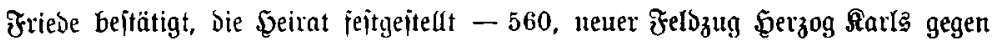

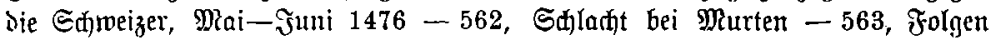
Der Shllabj: 3ujanmenbrud) ber burgumbifiten (Brobmadjt -567 , Stellung

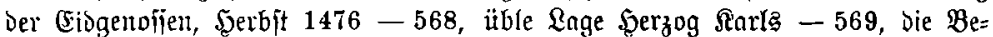

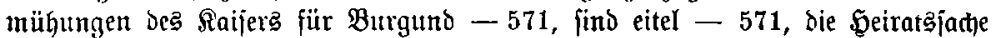
gefidjert -572 , Felszug ber Burgunder gegen Rothringen: S(f)(ad)t bor Rauch

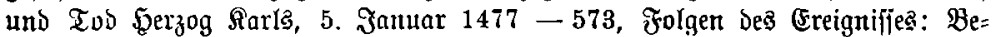
tvegung in ben Rieberlanden, Franfrein) gegen Burguno - 578, sie Eniferlidje

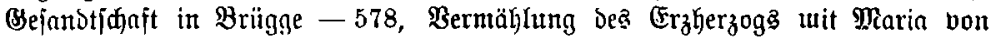

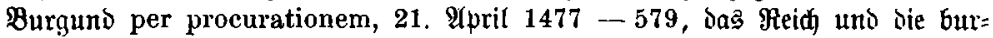

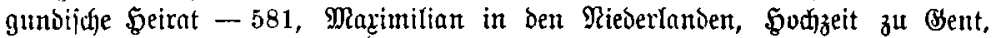
19. Augult 1477 - 582, Felbzug dea (5rzherzoge gegen frantreid) - 583 .

Dreiunbzwauzigftez Rapitel. S. 584-617. Der Raijer und Matthią Cor: biuus 1475-1177. Der neue Bund zrijalen Böhmen und dem Saijer. Des Raijers Rrieg mit Ungarn und jeine Folgen. Hugarn

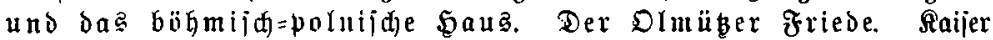
Fricorid uno Matthias (Sorbinus 1475-1477 - 586, Erzbifdof Johann von Bran fonmt nad) S̈fterreid - 586, Diplomatijder Arieg zıiifinen Dem Paijer und Ungarn - 589, innere Muruken in :̈fterreid -590 , ber Streit um Eroffen

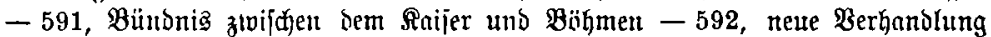
des Raijers ntit llugarn - 594, Sndjinge im Sijten zu Beginn 1477 - 596, Seerfahit ber Böhmen nad) D̈fterreid) - 598, Die Ungarn in S̈ (terreid) - 601,

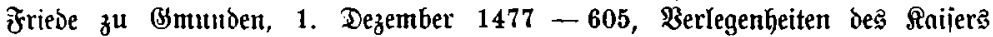
- 608, Stellung bes Ungarfb̈utg - 609, Friebenåbemilhungen im Streite um Bähmen, Berbartlungen z" Brünn uno Djen, März-Dftoher 1478-614, Rrieg zwijđen Ungarn unb Brantenburg -616 , ber Friedenātag jt Olmiï, Juli $1479-617$.

Bierunbzwanzigites Rapitel. S. 618-634. Die Anfridtung ber habz=

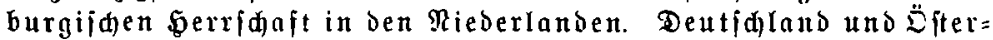
reid) it ber Türlennot 1477-1479. Wieberaugbrud bes Streite?

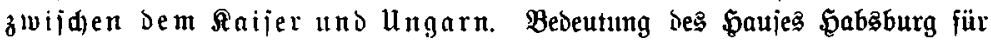

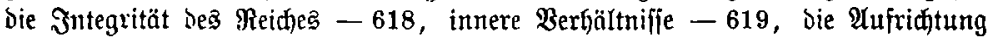
Der habsburgifdent ఏerrfdaft in Den Rieberlanden 1477 - 621, Der Stillitand bon Lets, 18. September 1477 - 622, Friede ztwijden Burgutd und ben Cis=

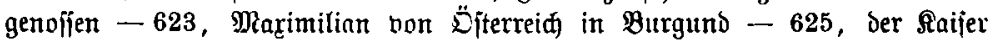
uno jein $S_{0 h n}$ in Burgund -626, bie $\mathfrak{B a f f e n r u h e ~ z u ~ D o u a y , ~ 1 6 . ~ J u n i ~}$ 1478 - 627, Mnximilians politijdes Snjtem -629, Lage ber öfterreidijđjen Erblanive $1478-631$, neuer Streit Dę Saijerß̄ mit Ungarn wegen ber Bran= Sal 2 burger Sađje - 634.

F̈unjunbzanzigites Sapitel. S. 634-674. Das beutide Reid und bie Rirfe vom zreiten $\mathfrak{S u j f i t t e n t r i e g e ~ b i a ~ z u m ~ S t r e i t e ~ u m ~ b a s ~ E r z = ~}$ bistum Salzburg. Saifer Friedrid) III. uno Siztus IV. Der Streit um die Rirden von Ronłanz, Mainz, Röln, Bajial und Salzburg. Das firdlid)e $\mathcal{L} e b e n$ in Deutidjlano von 1448-1490: Das reli=

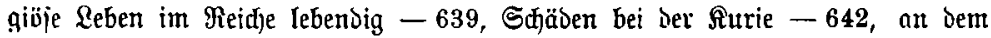

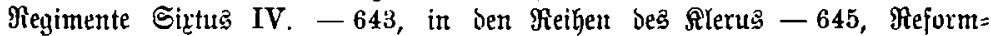
beriutue in Deutichlano - 649, ßapit Sirtus IV. uns Railer Frieorid - 653,

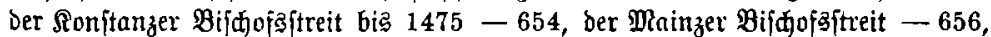
ber Streit um bie Ronftanzer Rirthe bis 2lugujt $1480-659$, der Streit um 


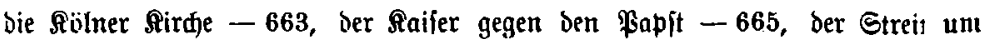

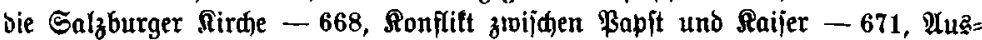

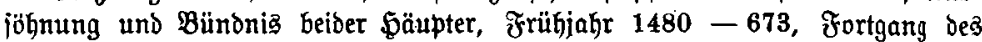
Salzburger Streitez, Ronflilt wegen der Baffauer Rirde - 674.

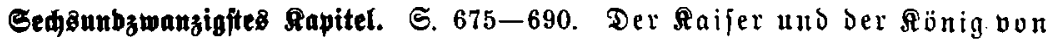
Ulngarn im Saljburger Rirdelltreite 1479-1480. Burguno und Franfieid. Die Reidstage bon 1479 und 1480. Der Salfburger Rirchenftreit fïhrt zum ßertwürfnis jwifłen ১em Raijer und Dem Ränig von

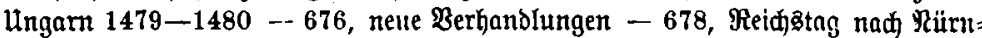

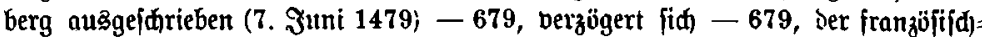

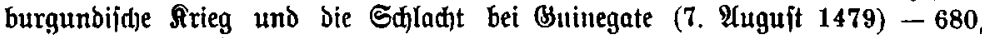

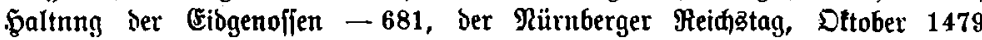

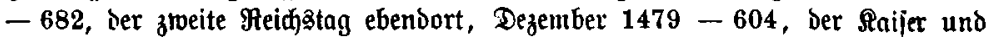

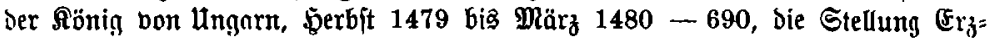
herzog Marimilans, F́nühjahr 1480, wièer ungünf́tiger -690 .

Siebenunbzwanzigftę Aapitel. S. 690-719. Die 9 ärnberger Reidstage von 1480 und 1481. Waffenruhe zivifden Frantreid unb burgund. Burgundifde Bläne. Fortgang bes Streites um Salzburg und

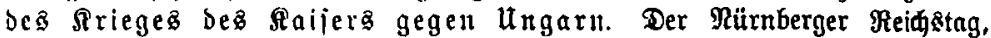

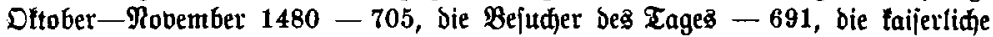
Bropofitiolt - 692, bie erite Belvilligung - 693, die "J̌urtidrittapartei" - 694, ber $\Re$ urfürit bon Branienburg gegen fie -694 , bie Berhandlungen utto $\mathfrak{B} e=$

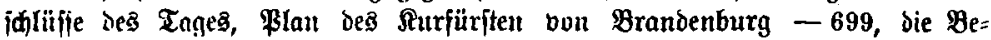
hauptungen Der Begner - 700, Berfaffungafragen - 701, bie Bejđlüfje des Tages - 707, bie Titufen in Otranto - 705, Erfolg Des ßeidątages - 706,

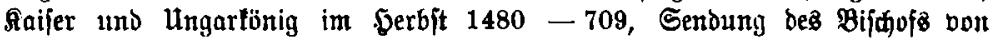

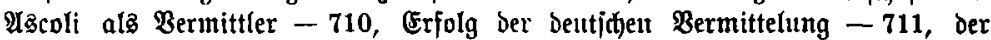

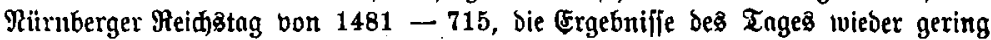

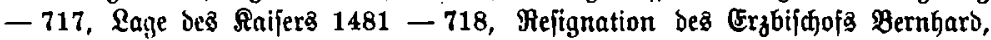
29. Nuvember $1481-718$, brobenber Ungarfrieg -719 .

Adtunbzwantigftes Rapiter. S. 720-740. Mitteleuropa in Jahre 1482. Bapft Sirtua IV. und bie legten $\mathfrak{J a h r e}$ feines $\mathfrak{W a l t e n} 3$. Marimilian bon D̈fterreid und bie Flanorer. Der Raifer im $\mathfrak{A}$ ampfe mit bem

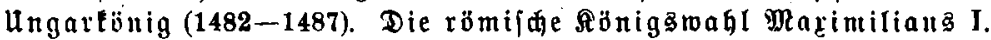

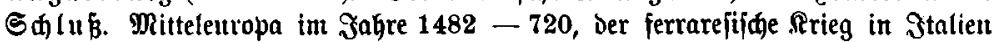

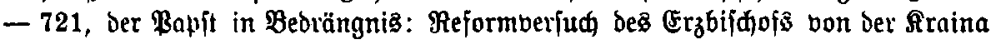

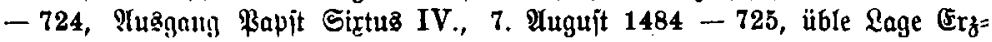

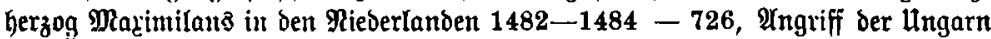
auf D̈fterreid) mito ibre Erfolge 1482-1484 - 737, neue Bermiltelungaberjud)e

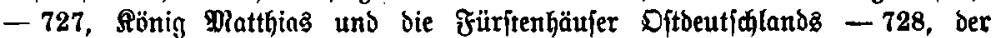

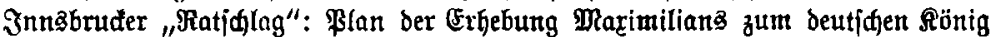
Februar 1484 - 731, Der Fürtitentog zll Franffurt, Sanuar $1485-733$, ber Surfürjt von Brandenburg gegen bie $\mathfrak{W a h l}-735$, neue Erfolge Jer Ungarn:

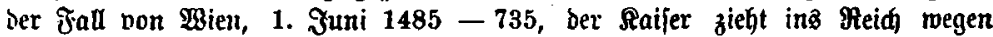

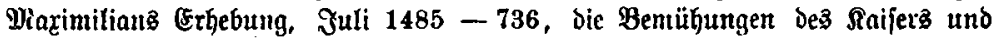

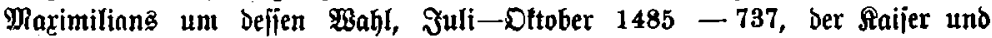

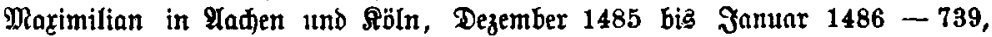
beibe am Surtage zu Frantfurt, Maximilian $\mathfrak{B a b l , ~ 1 6 . ~ F e b r u a r ~} 1486$ - 740 . Sd) 